Chirurg 2010 · 81:643-646

DOI 10.1007/s00104-010-1946-y

Online publiziert: 2. Mai 2010

(c) Springer-Verlag 2010

\author{
A.M. Mols ${ }^{1} \cdot$ S. Reiter-Theil ${ }^{2} \cdot$ D. Oertli ${ }^{1} \cdot$ C.T. ViehI ${ }^{1}$ \\ ${ }^{1}$ Viszeralchirurgie, Universitätsspital Basel \\ ${ }^{2}$ Fachbereich Medizin- und Gesundheitsethik, \\ Medizinische Fakultät, Universitätsspital Basel
}

\title{
Futility: ein Begriff im chirurgischen Alltag?
}

rückhaltend verhielt, wechselte die Familie kürzlich den Hausarzt.

Es besteht ein Status nach Sigmaresektion bei Sigmadivertikulitis vor Jahren, ein Status nach Resektionsrektopexie sowie Status nach Hysterektomie. Die Patientin leidet an einer Depression.

Aktuell bestehen abdominelle Schmerzen, zunehmende Appetitlosigkeit sowie einmaliges Erbrechen am Vortag. Der letzte Stuhlgang war vor 2 Tagen nach Verabreichung eines Einlaufes.

\section{Befund}

- Adipositas permagna, reizlose Narben, Meteorismus. Keine Klopf- oder Druckdolenz, kein Peritonismus. Darmgeräusche hochgestellt, klingend, normfrequent.

- Labor: makrozytäre Anämie, keine Erhöhung der Infektparameter.

- Röntgenübersicht des Abdomens: massive Koprostase, mehrere Dünndarmspiegel.

Auf unserer Notfallstation wird eine 94jährige Patientin durch ihre beiden Töchter vorgestellt. Nach Angaben der Angehörigen hat die Patientin seit Wochen abdominelle Beschwerden. Sie selbst kann aufgrund einer Demenz keinerlei Angaben zur Anamnese machen. Die Patientin lebt seit Jahren in einer Pflegeeinrichtung. Eine durch den Hausarzt veranlasste Koloskopie vor wenigen Monaten ergab keinen pathologischen Befund bei allerdings mangelnder Beurteilbarkeit aufgrund von Stuhlverschmutzungen. Da sich der Hausarzt bezüglich weiterer Abklärungen zu-

\section{Diagnose}

Aufgrund der Befunde stellen wir die Diagnose eines Subileus.

\section{Verlauf}

Wir führen über mehrere Tage eine konservative Therapie mit abführenden Maßnahmen und Flüssigkost durch; die Patientin erhält eine analgetische und antiemetische Medikation. Bei zunehmender Aggressivität der dementen Patientin wird ein gerontopsychiatrisches Konsilium und, den Empfehlungen entsprechend, eine neuroleptische Therapie eingeleitet. Die Patientin wehrt Ärzte und Pflegende mit wechselnder Intensität $a b$, teilweise unter Verwendung deutlicher Kraftausdrücke.

Über mehrere Tage zeigt sich ein erfolgreiches Ansprechen auf die abführenden Maßnahmen. Mehrmalig kommt es zu Beginn der Behandlung zum Absetzen großer Stuhlportionen, bei vorsichtigem Kostaufbau tritt dann erneut ein zunehmender Stuhlverhalt auf. Am 6. Tag der Hospitalisation setzt schwallartiges Erbrechen ein; es sind nun hochgestellte Darmgeräusche auszukultieren. Wir stellen die Diagnose eines konservativ nicht beherrschbaren Ileus.

Die Patientin besitzt keine Patientenverfügung. Die Töchter der Patientin können keine Auskunft über den mutmaßlichen Patientenwillen geben. Gespräche mit der Mutter vor Beginn ihrer demenziellen Entwicklung über Entscheidungen am Ende des Lebens hat es nicht gegeben.

Eine der beiden Töchter wünscht, dass „alles“ für ihre Mutter getan wird, diese Tochter hatte den Hausarztwechsel initiiert. Die zweite Tochter äußert sich in Anbetracht des Alters und der Gesamtsituation der Patientin zurückhaltend, scheint allerdings mit der Situation emotional und intellektuell überfordert zu sein. Wir haben keinen Anhalt dafür, welche der Töchter das vertrautere Verhältnis zur Mutter hatte und möglicherweise eher den Patientenwillen widerspiegelt. 
Bei nicht bekanntem Patientenwillen stellt sich nun also die Frage, ob wir eine Operation des Ileus für sinnvoll erachten. Aus chirurgischer Sicht besteht bei konservativ nicht beherrschbarem Ileus klar die Indikation zum operativen Eingriff. Wir sehen uns jedoch konfrontiert mit einer über 90-jährigen schwer dementen Patientin, die unselbständig in einem Pflegeheim lebt, depressiv und abwehrend ist.

Darf eine über 90-jährige demente $\mathrm{Pa}$ tientin nicht an einem Ileus sterben? Verlängern wir mit einer Operation ein Leben, dessen Lebensqualität wir nicht klar beurteilen können? Können wir die Risiken einer Laparotomie bei der Hochbetagten mit möglichen Komplikationen in Kauf nehmen?

Aber: Sind wir als Chirurgen nicht fast gezwungen, einen eindeutigen Ileus operativ zu beheben? Dürfen und wollen wir einen Menschen im Ileus sterben lassen, und wenn ja, unter welchen Bedingungen ist dies vertretbar?

Dies ist ein Fall, dessen ethische Herausforderung im klinischen Alltag nicht außergewöhnlich ist. Obwohl oder gerade weil dieser Fall so „gewöhnlich“ ist und uns in anderen Spielarten so häufig konfrontiert, haben wir versucht, die Schwierigkeit der Indikationsstellung aufzuarbeiten.

\section{Theoretischer Hintergrund - Begriffsdefinition Futility}

Futility, zu Deutsch „Sinnlosigkeit“, „Zwecklosigkeit“ ist ein komplexer Begriff der Medizinethik, der Übertherapie zu beschreiben versucht. In einer sehr weiten Auslegung des Begriffes geht es um Situationen, in welchen prognostiziert werden kann, dass eine Therapie den Zustand des Patienten nicht verbessert und daher unterlassen werden soll.

Die klare Eingrenzung des Begriffes ist erheblich schwieriger. In den 1980er Jahren kam der Begriff Futility zunächst in den USA auf, da zunehmend Fälle gesehen wurden, bei denen Patienten und Angehörige Therapien forderten, die Ärzte nicht als sinnvoll erachteten. In dieser ersten Phase der Futility-Debatte gingen Reaktion und Gegenreaktion auf eine verän- derte ärztliche Rolle und die Eingrenzung des medizinischen Paternalismus ein [5].

Schneiderman et al. versuchten, den Begriff auf einen kleinsten gemeinsamen Nenner zu bringen: Eine Therapie sei "quantitativ futile“, wenn eine Wahrscheinlichkeit (aus Daten und Erfahrung) unter $1 \%$ besteht, dass eine Behandlung Erfolg hat, sie sei „qualitativ futile“, wenn die Behandlung an Bewusstlosigkeit oder Abhängigkeit von intensivmedizinischer Behandlung nichts ändern kann [11].

Eine Welle der Kritik bezog sich sowohl auf die quantitative $1 \%$-Grenze, als auch auf die Eingrenzung der qualitativen Futility, da sie sich auf ein sehr enges $\mathrm{Pa}-$ tientenspektrum - intensivmedizinische Fälle - bezieht. Auf Patienten in Pflegeeinrichtungen oder Patienten mit schweren Behinderungen, die außerhalb der Intensivstationen häufig erhebliche Probleme bei Indikationsstellungen bereiten, wurde nicht eingegangen. Dennoch wurde als hilfreich erachtet, dass diese Begriffsklärung eine Beurteilung des medizinischen Erfolges sowie eine Bewertung der zu erwartenden Lebensqualität ermöglicht [2, 3].

In der weiteren Diskussion wurde fortan weniger versucht, eine Definition des Begriffes zu etablieren, da er sich offensichtlich als zu schwer fassbar herausstellte. Es wurde vielmehr daran gearbeitet, dessen Operationalisierung in institutionellen und legislativen Maßnahmen zu erreichen.

Dabei ging und geht es vor allem in den USA weiterhin in erster Linie um Konflikte zwischen Behandelnden und Angehörigen in der Therapie Schwerstkranker. Eine gegenwärtige Entwicklung im Umgang mit Futility bezieht sich vor allem auf die Kommunikation mit Angehörigen und auf eine Akzeptanz von unterschiedlichen Werten am Ende des Lebens $[7,9]$.

Herauszustellen ist, dass das Konzept „Futility“ nicht mit einer Diskussion um Ressourcenverteilung interferieren darf. Futility ist ein Begriff der Medizinethik, der die Angemessenheit medizinischer Maßnahmen in Bezug auf das Individuum bezogen bewertet [8].

\section{Anwendung der Theorie im konkreten Fall}

Wir sehen hier also einen Begriff, von dem wir zunächst intuitiv annehmen, dass er unser ethisches Problem passend als Übertherapie beschreibt, der allerdings aufgrund seiner vagen Definition für unseren Fall schwer anwendbar bleibt.

Dennoch kommen wir in der Auseinandersetzung zu einem der Kernpunkte: Welche Intention verfolgen wir hier mit einem operativen Eingriff? Natürlich scheitern wir mit einer Intention, die auf eine Wiederherstellung der allumfassenden Gesundheit der Patientin abzielt. Dies ist unmöglich, die Patientin wird schwer dement, depressiv und pflegeheimabhängig bleiben.

Unsere Intention kann lediglich die Vermeidung eines - möglicherweise qualvollen Todes im Ileus sein und dient daher einer palliativen Zielsetzung. Palliative care "provides relief from pain and other distressing symptoms; affirms life and regards dying as a normal process; intends neither to hasten nor to postpone death ...", so beschreibt es die WHO [6]. Damit nehmen wir als vorrangige Verpflichtung die Schadensvermeidung („nonmaleficence“) an; diese kann ggf. mit dem Respekt vor Autonomie (bzw. den Wünschen von Patient oder Angehörigen) in Konflikt treten.

Obwohl wir weiter durchaus Bedenken haben, die alte Dame zu operieren, sehen wir in unseren Überlegungen die Vermeidung von Leid am ehesten durch die Vermeidung eines Todes im Ileus gewährleistet. Wir entscheiden uns daher für eine Operation.

Intraoperativ sehen wir eine fortgeschrittene Peritonealkarzinose bei unklarem Primärtumor. Es wird eine Jejunotransversostomie angelegt. Die Patientin erholt sich rasch von dem Eingriff. Allerdings kommt es postoperativ zu einer zunehmenden Nahrungsverweigerung der Patientin. Im Lichte der Gesamtsituation nehmen wir dies als Hinweis auf den mutmaßlichen Willen der Patientin und entscheiden im Einvernehmen mit den Töchtern gegen die parenterale Ernährung oder Sondenkost. Wichtig ist hier, dass die (eigenen) Präferenzen von Angehörigen zwar gehört, aber nicht automatisch 
mit dem (mutmaßlichen) Patientenwillen (und dem wohlverstandenen Patienteninteresse) gleichgesetzt werden [7, 9]. Die Patientin wird am 12. postoperativen Tag in ihr Altersheim zurückverlegt.

\section{Warum ist eine}

\section{Auseinandersetzung mit der} ethischen Dimension notwendig?

Wir sehen verschiedene Gründe, warum wir eine Auseinandersetzung mit der ethischen Problematik bei Indikationsstellungen bei Patienten, die sich nicht äußern können, für unbedingt erforderlich halten.

Primär geht es um eine optimale Versorgung der Patienten. In Fällen wie dem unseren bedeutet dies leider häufig nicht nur primum nihil nocere, sondern - pointierter - ein Abwägen, welche Maßnahme am wenigsten schadet. Oftmals werden in ethisch schwierigen Situationen - je nach Fachgebiet und persönlichem Standpunkt - fast schon reflexartig Meinungen laut wie: „über einem Ileus darf die Sonne weder auf- noch untergehen“ vs. „ein alter Mensch muss auch sterben dürfen". Wichtig ist, dass wir nicht die Chance verpassen, eine Lösung zu finden, die für den Patienten die beste Alternative darstellt, unabhängig von unseren persönlichen Wertvorstellungen.

Zweitens müssen wir den Angehörigen vermitteln können, dass wir überlegte und auf die Patienten zugeschnittene Lösungen anbieten. In unserem dargestellten Fall konnten wir die Töchter aufgrund deren Uneinigkeit nicht an einer Entscheidung beteiligen. Eine solche Beteiligung sollte jedoch nach Möglichkeit angestrebt werden [9].

Wenn man sich vor Augen führt, wie schwer dieser Fall aus Sicht der nächsten Angehörigen erlebt wird, wie viel Leid die Töchter bei ihrer Mutter erfahren, wie Angehörige selbst in einer solchen Entscheidungsfindung zerrissen sein können, so haben wir als behandelnde Ärzte die Pflicht, den Angehörigen unsere Intentionen und die Indikationen in angemessener Form mitzuteilen - jenseits von „Sprechblasen“.

Drittens sind wir es uns auch selbst schuldig, in ethischen Grenzfällen unsere Motive herauszuarbeiten. Operationen

Chirurg 2010 -81:643-646 DOI 10.1007/s00104-010-1946-y

(c) Springer-Verlag 2010

A.M. Mols · S. Reiter-Theil · D. Oertli · C.T. Viehl

Futility: ein Begriff im chirurgischen Alltag?

\section{Zusammenfassung}

Ethisch anspruchsvolle Indikationsstellungen bei Patienten, die ihren Willen nicht selbst äußern können, wie beispielsweise schwer demenzkranke Patienten, konfrontieren uns häufig. Es stellt sich hierbei die Frage, ob wir mit operativen Eingriffen eine Übertherapie vollziehen. Der Begriff, "futility" der Medizinethik beschreibt Übertherapie, ist allerdings für eine konkrete Anwendung problematisch, da eine exakte Definition fehlt.

In der klinischen Situation von schwer demenzkranken, hochbetagten Patienten muss in chirurgischen Abteilungen eine Aufarbeitung von medizinischem Hintergrund, Lebensumständen des Patienten und belegtem

\section{Futility: a concept in routine surgery?}

\section{Abstract}

In surgical practice we are often confronted with ethically challenging situations when treating patients not capable of expressing their own wishes. Issues of futile treatment by indicating operations arise particularly with regard to severe dementia. The concept of futility describes forms of therapy which are not appropriate to improve the patient's condition, but for application in clinical practice the concept is insufficiently defined.

In ethically challenging situations, e.g. in the treatment of severely demented patients, we need to balance the medical condition and prognosis with the documented or as- oder mutmaßlichem Patientenwillen erfolgen mit dem Ziel, Indikationen individualisiert zu stellen. Nur so können diese Patienten optimal versorgt werden, eine klare Kommunikation über Behandlungsziele mit Angehörigen stattfinden sowie eine Vermeidung eines „burn out" bei den Behandelnden erreicht werden. Von großem Nutzen ist hierbei eine enge Zusammenarbeit mit Medizinethikern.

\section{Schlüsselwörter}

Medizinethik · Übertherapie · Patientenwille . Indikationsstellung · Chirurgie sumed wishes of the patients. Involving the relatives competently is essential. The indication for surgery in patients with severe dementia, for example, needs to be individualized striving for optimal care, a clear communication about treatment goals with the relatives and preventing distress and burnout for staff. Co-operation with specialists in medical ethics is recommended.

\section{Keywords}

Medical ethics - Futility - Patient's wishes . Indications · Surgery 
bei schwer Demenzkranken sind für uns als Operateure belastend. Das Bewusstsein von Futility oder Übertherapie ist für denjenigen, der eine Operation vornimmt, moralisch kaum tragbar. Studien belegen einen Zusammenhang zwischen der Wahrnehmung von Übertherapie und einem erhöhten Risiko für Distress oder Burnout [4].

Wir empfehlen bei Indikationsstellungen, die ethisch problematisch erscheinen, eine klare und möglichst strukturierte Vorgehensweise.

Zentrale Punkte der Beurteilung sind:

- Krankheitsverlauf und zu erwarten-

des operatives Outcome,

- aktuelle Lebenssituation des Patienten,

- direkt geäußerter oder mutmaßlicher Patientenwille, der durch die Angehörigen oder zusätzlich den Hausarzt erfragt werden sollte.

Als äußerst hilfreich erachten wir hierbei eine enge Zusammenarbeit mit der Medizinethik, welche an vielen Kliniken ethische Konsilien anbieten. Im Universitätsspital Basel ist zudem ein strukturiertes Gespräch der Behandelnden (ethische Fallbesprechung) unter Zuhilfenahme der Leitlinie METAP (Modular Ethical Treatment Allocation Process) etabliert [7, 10]. Wir als chirurgische Abteilung haben positive Erfahrungen in der Zusammenarbeit sammeln können, da uns das strukturierte Angehen der ethischen Fragestellungen und der unvoreingenommene Blick von außen oftmals eine zusätzliche Klarheit bringt [1]. Eine systematische Evaluation ist im Gange ${ }^{1}$.

\section{Fazit}

Operationen bei Patienten, die ihren Willen nicht äußern können und insbesondere hochbetagte, demenzkranke Patienten wie in unserem Fall, stellen eine Herausforderung in der Indikationsstellung dar. Diese Indikationsstellungen

\footnotetext{
1 SNF Project: No. 32003B-125122 ("Fairness and Transparency. Clinical Ethical Guideline METAP - Evaluation, Methodological Foundation and Improvement", 2009-2011); Principal Investigator: Prof. Dr. S. Reiter-Theil, CoInvestigator: Prof. Dr. H. Pargger; Clinical Partner: Department of Surgery, USB, Basel.
}

müssen unter Kenntnisnahme aller medizinischen wie auch aller sozialen Umstände und möglicher Erfolgsaussichten gefällt werden. Von großer Bedeutung ist die kompetente Einbeziehung der Angehörigen. Insbesondere ist der mutmaßliche Patientenwille von Präferenzen der Angehörigen zu unterscheiden. Wichtig erscheint es uns, dass innerhalb chirurgischer Abteilungen eine bewusste Auseinandersetzung mit ethischen Problemstellungen stattfindet, um Patienten, Angehörige und uns selbst vor vorgefertigten Meinungen zu schützen. Hilfreich ist hierbei eine Reflexion der Intentionen verschiedener Behandlungsformen im Detail. Der Begriff Futility ist als solcher nur schwer anzuwenden. Im Vordergrund sollte die explizite Begründung der Maßnahmen, auch in ethischen Begriffen, stehen, um mögliche Rationalitätslücken zu schließen. Empfehlenswert ist eine enge Zusammenarbeit mit der Medizinethik.

\section{Korrespondenzadresse \\ Dr. A.M. Mols}

Viszeralchirurgie, Universitätsspital Basel, Spitalstrasse 21, 4031 Basel,

Schweiz

molsa@uhbs.ch

Interessenkonflikt. Der korrespondierende Autor gibt an, dass kein Interessenkonflikt besteht.

\section{Literatur}

1. Albisser Schleger $\mathrm{H}$, Pargger $\mathrm{H}$, Reiter-Theil $\mathrm{S}$ (2008) Futility - Übertherapie am Lebensende? Gründe für ausbleibende Therapiebegrenzung in Geriatrie und Intensivmedizin. Z Palliativmed 9:67-75

2. Bernat JL (2005) Medical Futility. Definition, Determination, and Disputes in Critical Care. Neurocrit Care 2:198-205

3. Burns JP, Truog RD (2007) Futility. A concept in evolution. Chest 132:1987-1993

4. Meltzer LS, Huckabay LM (2004) Critical care nurses' perceptions of futile care and its effects on burnout. Am J Crit Care 13:202-208

5. Moratti S (2009) The development of "medical futility ": towards a procedural approach based on the role of the medical profession. J Med Ethics 35:369-372

6. National cancer control programmes (2002) policies and managerial guidelines, 2nd edn. WHO online

7. Reiter-Theil S (2008) Ethikberatung in der Klinik ein integratives Modell für die Praxis und ihre Reflexion. Ther Umsch 65:359-365

8. Reiter-Theil S, Albisser Schleger H (2007) Alter Patient - (k)ein Grund zur Sorge? Notfall Rettungsmed 10:189-196
9. Reiter-Theil S, Mertz M, Meyer-Zehnder B (2007) The complex roles in end-of-life decision-making: An ethical analysis. HEC Forum 19:341-364

10. Reiter-Theil S, Mertz M, Meyer-Zehnder B et al (2010) Klinische Ethik als Partnerschaft - oder wie eine ethische Leitlinie für den patientengerechten Einsatz von Ressourcen entwickelt und implementiert werden kann. Ethik Med (im Druck)

11. Schneiderman LJ, Faber-Langendoen $K$, Jecker N (1994) Beyond futility to an ethic of care. Am J Med 96:110-114 